Presented at the International Workshop on Accelerator Alignment (IWAA'99), October 18-22, 1999, Grenoble, France

\title{
Long-Term Stability of the APS Storage Ring*
}

\author{
H. Friedsam, M. Penicka, J. Error \\ Argonne National Laboratory, Argonne, Illinois, USA
}

\section{INTRODUCTION}

The Advanced Photon Source (APS), a third-generation synchrotron light source, was commissioned in 1995 at Argonne National Laboratory and has been fully operational for beamline users since 1997. The APS storage ring can accommodate up to 68 user beamlines (Figure 1); about $70 \%$ of the available beamlines are currently in use by various collaborative access teams (CATs). The 7-GeV synchrotron light source produces light in the soft to hard x-ray range that is used for research in such areas as x-ray instrumentation; material, chemical and atomic sciences; biology; and geo/soil/ environmental sciences [1].

For the successful operation of an X-ray light source such as the Advanced Photon Source, the longterm stability of the concrete floor supporting the beam components and user beamlines is crucial. Settlements impact the orbit and location of the X-ray source points as well as the position of the x-ray beamlines.

This paper compares the results of two successive resurveys of the APS accelerator components performed in 1995 and 1998.

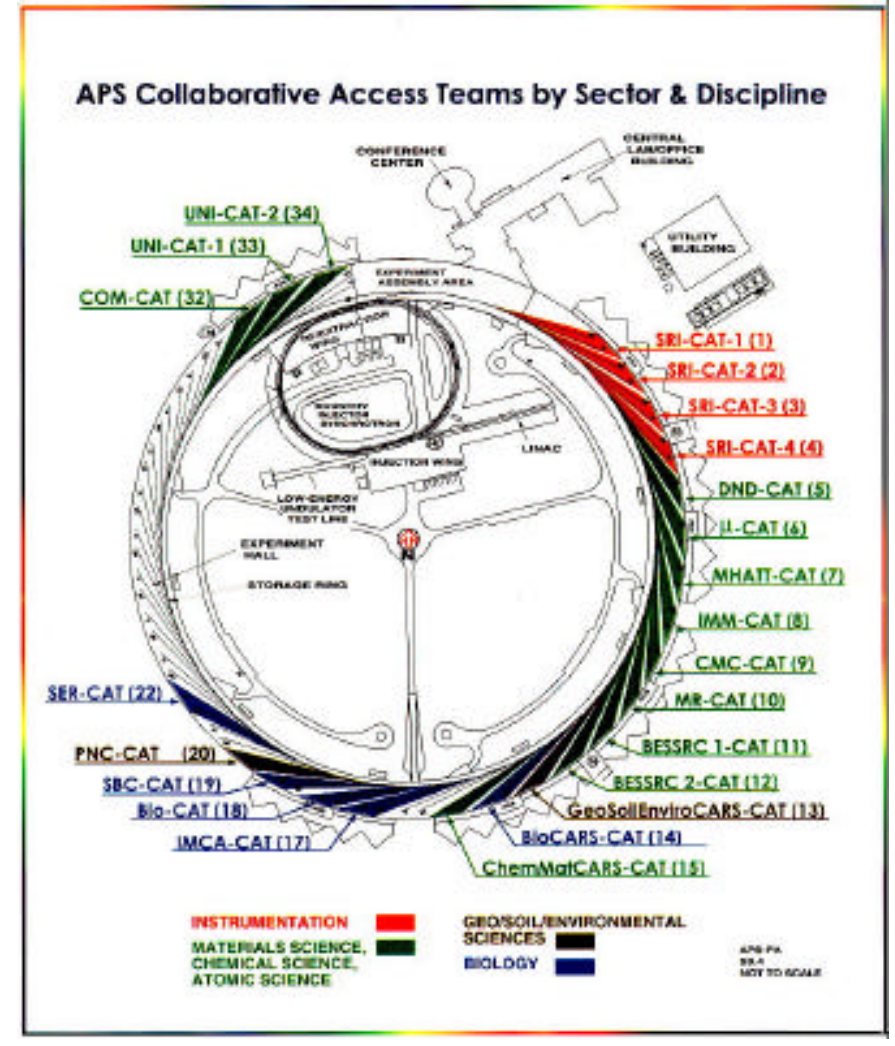

Fig. 1 APS Sector Layout

\footnotetext{
* Work supported by U.S. DOE, Office of Basic Energy Sciences, under Contract No. W-31-109-ENG-38.
} 


\section{ALIGNMENT TOLERANCES}

During construction of the APS the Survey and Alignment Group placed the accelerator components within tight absolute and relative tolerances derived from lattice simulations performed by the APS Accelerator Physics Group. The tolerances for these components, reported in detail in previous IWAA proceedings such as IWAA93 [2], are summarized below.

\subsection{Absolute positioning tolerances}

The global placement tolerance requires the positioning of each beam component, in the APS reference frame, to within a vertical and horizontal envelope of $\pm 5 \mathrm{~mm}$ of the ideal position. The roll of all multipoles and dipoles has to be set to within $\pm 0.5 \mathrm{mrad}$.

\subsection{Relative positioning tolerances}

The relative alignment between beam elements depends on the type of component and its location in the accelerator system. The most stringent requirements have to be achieved for the positioning of the storage ring multipoles. According to the design specifications for those magnets [3], a relative alignment tolerance of $\pm 0.15 \mathrm{~mm}$ is required in both the vertical and horizontal directions.

\section{ALIGNMENT RESULTS}

After the completion of the storage ring girder alignment in early 1995, the positions of all multipoles and dipoles were measured once more using laser trackers [4]. In order to obtain the connection between individual tracker setups, additional temporary control points were installed and measured. The data, including elevation information, were analyzed using a bundle adjustment routine developed at Stanford Linear Accelerator Center (SLAC). The differences between the resulting magnet positions from the bundle adjustment and the ideal locations were then subjected to a smooth curve fitting operation to obtain an estimate for the achieved relative alignment [5].

The results of this smoothing process are shown in Figures 2 through 5 for the transverse and vertical deviations, respectively. One can see that the smooth curve fits the data points, which represent the deviations between the measured and ideal locations of each magnet fiducial, very closely. Each histogram shows the distribution of the point offsets from the fitted curve sorted in $0.1 \mathrm{~mm}$ wide bins. These indicate that the positioning of the components is well within the required placement tolerances. The standard deviation of the offsets from the point location to the smooth curve is $\pm 0.09 \mathrm{~mm}$ for the transverse position and $\pm 0.07 \mathrm{~mm}$ for the vertical position, respectively. The mean is zero in both cases. In addition, each histogram contains the Gaussian distribution for the achieved standard deviation as a solid line and the expected distribution using the given tolerance limits as a dashed line. All data points are within the $2 \sigma$ range of $\pm 0.3 \mathrm{~mm}$. 


\subsection{Data set from 1995}

P-Curve Smoothing for X Component

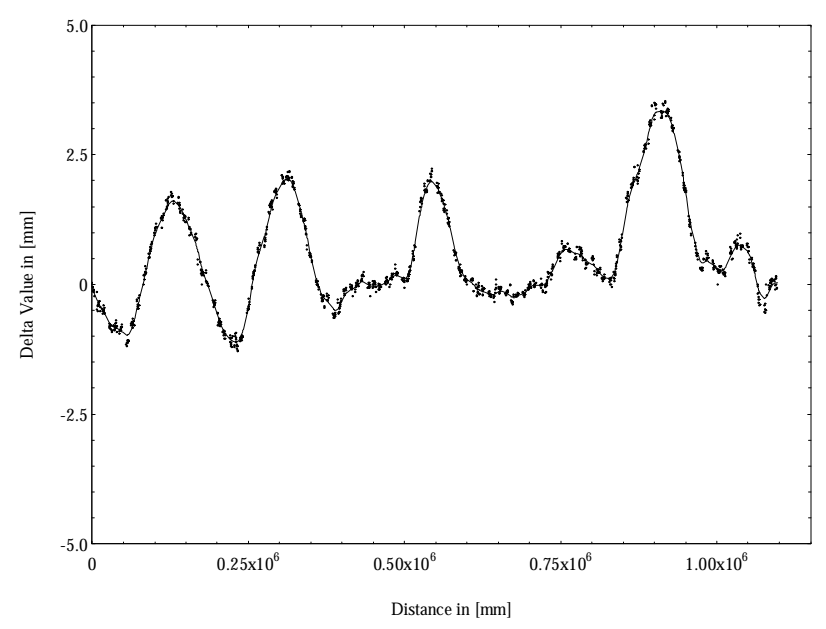

Fig. 2 Transverse displacements of the storage ring multipoles

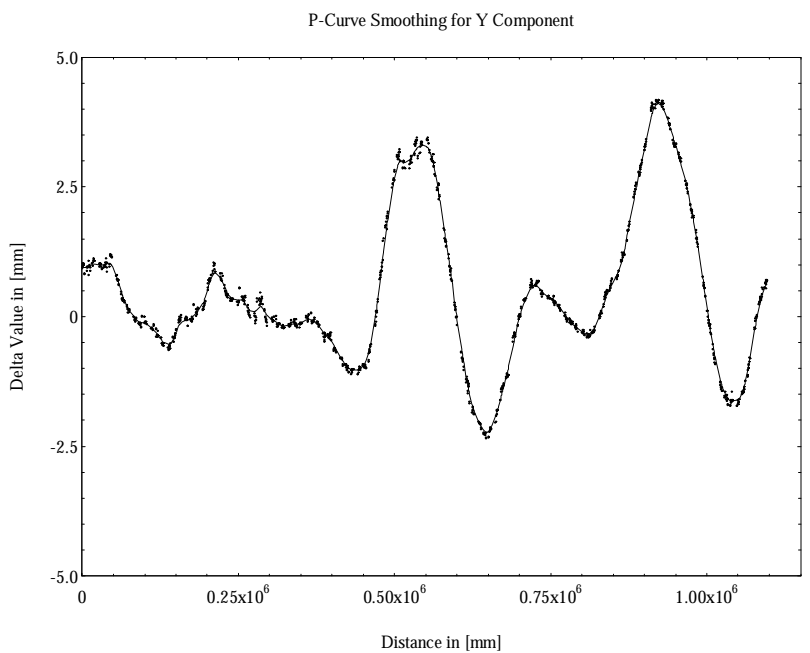

Fig. 4 Vertical displacements of the storage ring multipoles

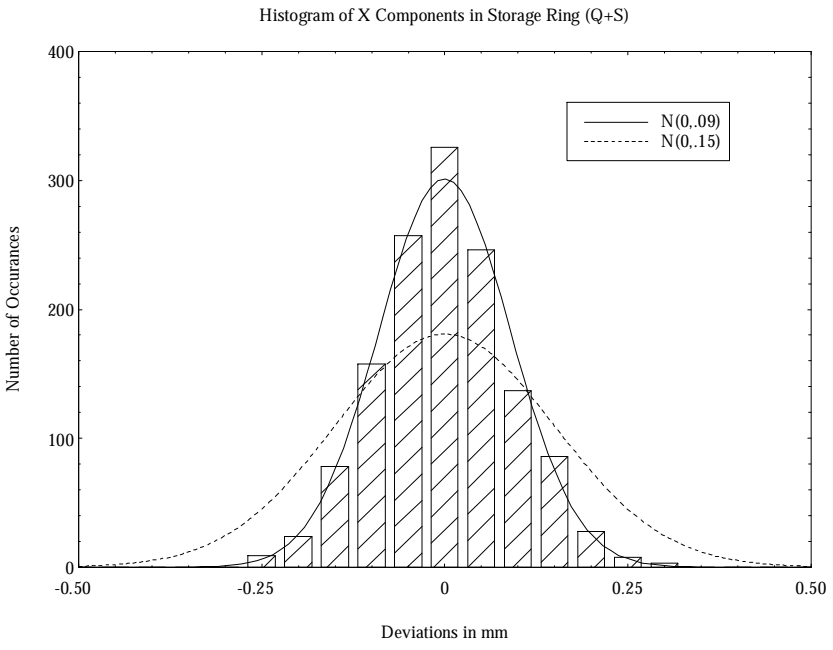

Fig. 3 Histogram of the transverse displacements of the storage ring multipoles

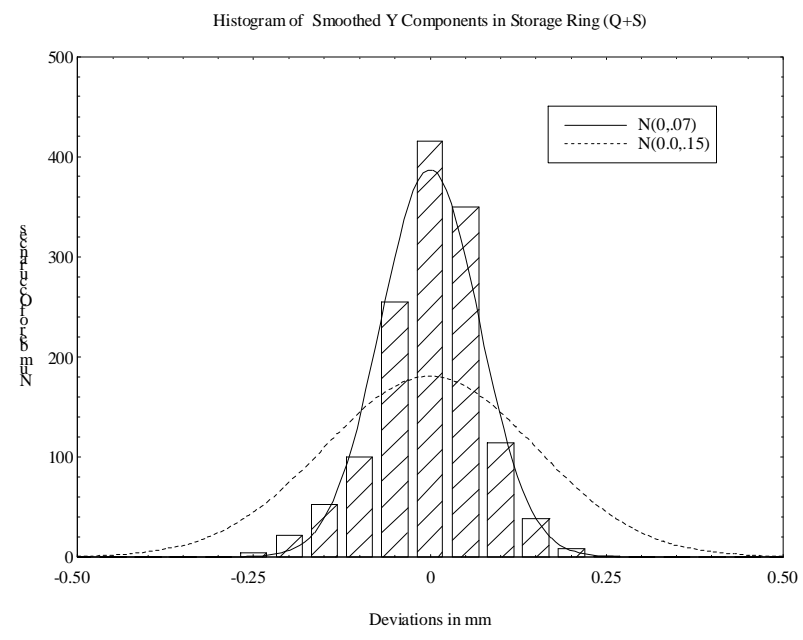

Fig. 5 Histogram of the vertical displacements of the storage ring multipoles

Since the APS operation schedule provides limited access for maintaining the accelerator, three years passed before we were able to perform a full resurvey of the APS storage ring. During a four-week shutdown in October of 1998 the entire storage ring was mapped a second time using two laser tracker systems. In successively shorter shutdowns, elevation data were collected to supplement the full data set. The results of this effort are shown in Figures 6 through 9. 


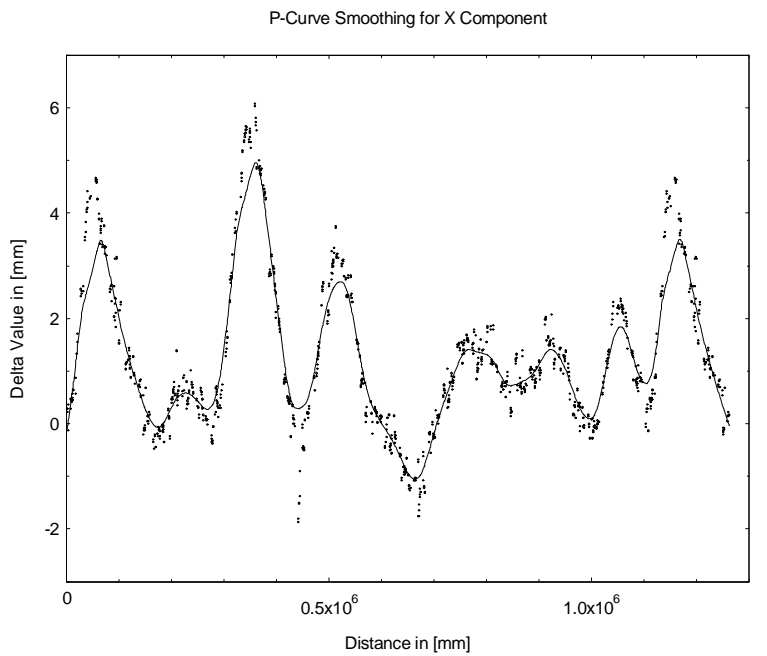

Fig. 6 Transverse displacements of the storage ring multipoles

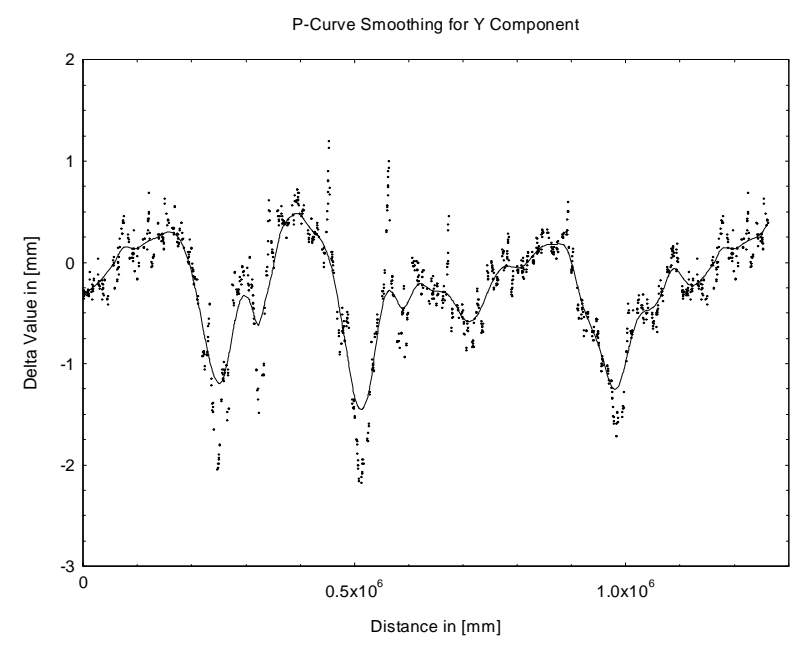

Fig. 8 Vertical displacements of the storage ring multipoles

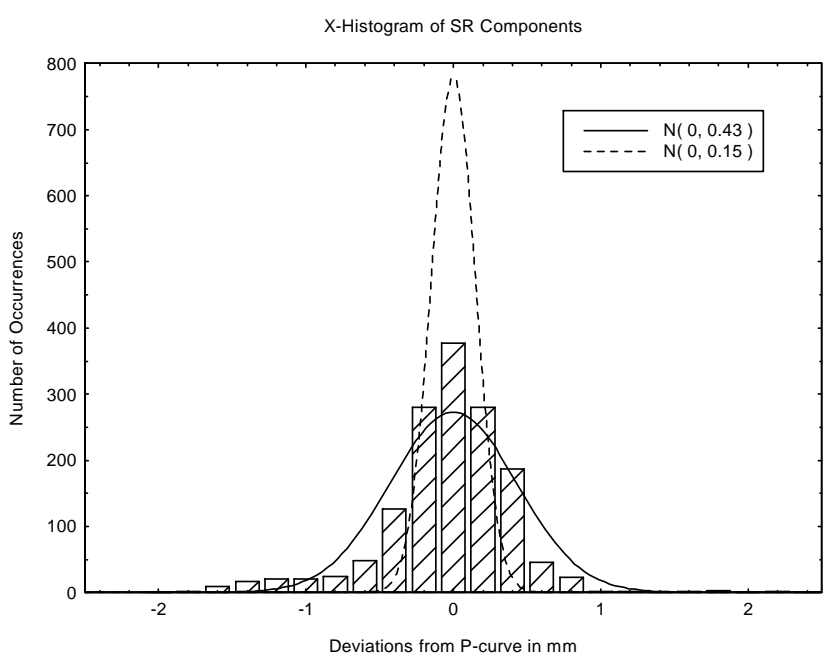

Fig. 7 Histogram of the transverse displacements of the storage ring multipoles

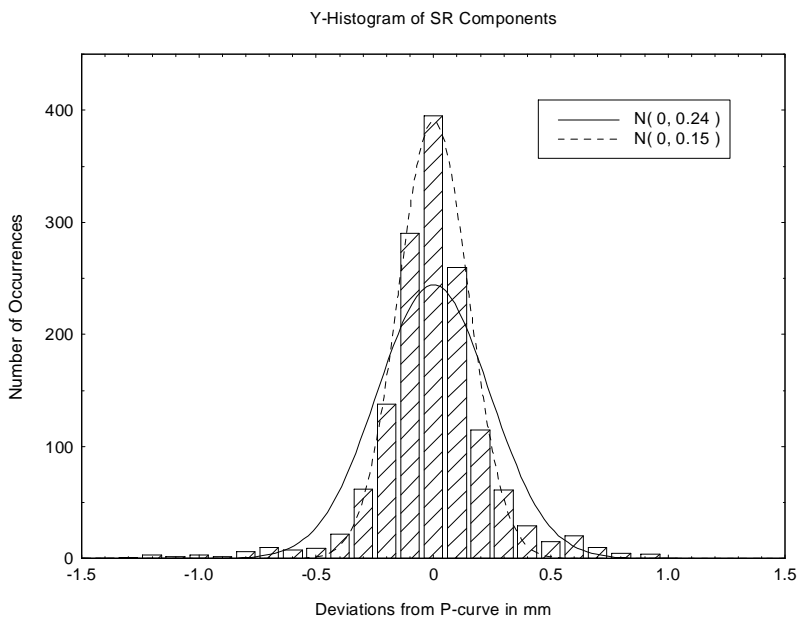

Fig. 9 Histogram of the vertical displacements of the storage ring multipoles

In comparison to 1995, the 1998 data set shows a deterioration of the APS storage ring alignment. The standard deviation for the relative transverse position changed by a factor of five and is now $\pm 0.43 \mathrm{~mm}$, while the standard deviation for the vertical relative positioning increased by a factor of three to $\pm 0.24 \mathrm{~mm}$. Surprisingly, the deterioration in the transverse direction is larger than in the vertical direction. This is in contrast to the common assumption that vertical settlements would be larger than transverse motions. 
It is apparent that the 1998 data set no longer meets the initial tolerance specifications, and a large fraction of the components falls outside the $2 \sigma$ limit. However, the operation of the accelerator so far has not suffered from this fact. Unfortunately, a direct comparison of the vital accelerator data between the 1995 and 1998 survey data sets is not possible due to the fact that many of the machine measurement tools were not available during the initial 1995 commissioning phase.

Since 1995 only two local realignments were necessary. The first was an experiment to evaluate the effects of a vertical girder realignment in selected sectors of the storage ring on beam operation parameters. The two sectors with the largest settlement were realigned using a smooth-curve-fitting routine to eliminate the largest settlement distortion. Adjustments up to $2 \mathrm{~mm}$ were required for this. However, the impact on the performance of the APS storage ring was negligible and hardly detectable with the measurement tools at hand [6].

The beam path in the area of the deformation was smooth before the realignment took place. This supports the choice of using a monolithic storage ring floor for construction. The floor does not permit any expansion joints that would cause discontinuities between adjacent slabs. Instead, a local deformation is being distributed over a large area, producing a smooth deformation [7].

The second modification required the artificial transverse distortion of two sectors of the APS storage ring for the purpose of implementing a new feedback system [8]. After the new lattice was realized, no major orbit distortions outside that region were measured. This indicates that as long as the particle beam follows a smooth beam path, the performance of the system will hardly change. However, it is very important to check for large steps between adjacent multipoles that have an impact on the beam emittance.

Comparing the two data sets one can see that the 1998 data contain string-like features that can only be explained by the displacement of an entire girder, indicating the possibility that the intra-girder alignment is more stable over time than the inter-girder alignment. In order to prove this hypothesis, the 1995 and 1998 data were each split into two parts. The first part analyzes the steps between adjacent multipoles on straight girders, while the second part examines the transverse and vertical steps between adjacent girders. Steps are simply calculated as transverse and vertical differences between adjacent components. 


\subsection{Intra-Girder Alignment Comparison}

The results of this process for the intra-girder alignment are shown in Figures 10 through 13 for the transverse and vertical steps of the 1995 and 1998 data. Each histogram shows the steps sorted in bins $0.1 \mathrm{~mm}$ wide. One can see that the standard deviation between these two data sets hardly changes and that most of the components are within the $2 \sigma$ limit, indicating that the alignment of the magnets on a girder is fairly stable over time as stated in the hypothesis. The standard deviation in the transverse case changes from $\pm 0.07 \mathrm{~mm}$ to $\pm 0.10 \mathrm{~mm}$, while in the vertical case it changes from $\pm 0.05 \mathrm{~mm}$ to $\pm 0.10 \mathrm{~mm}$.

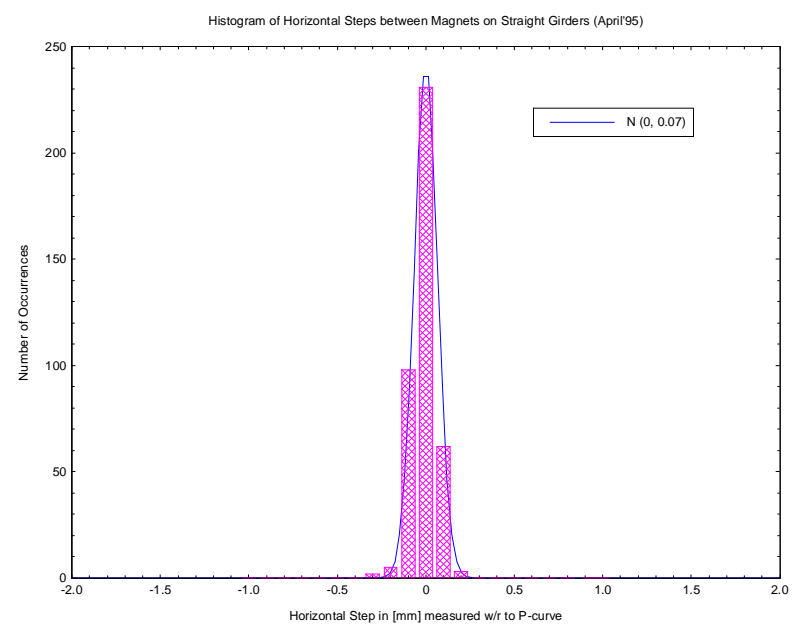

Fig 10 Transverse magnet step data, 1995

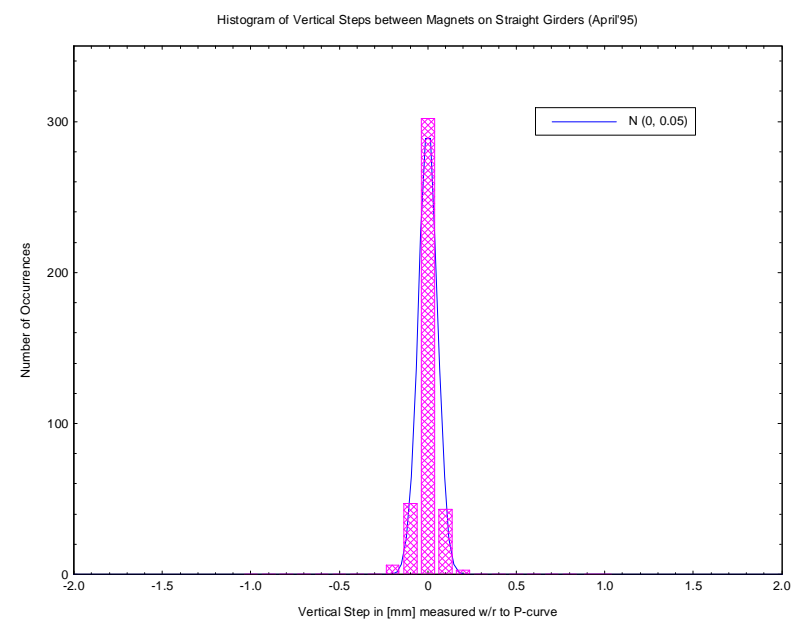

Fig 12 Vertical magnet step data, 1995

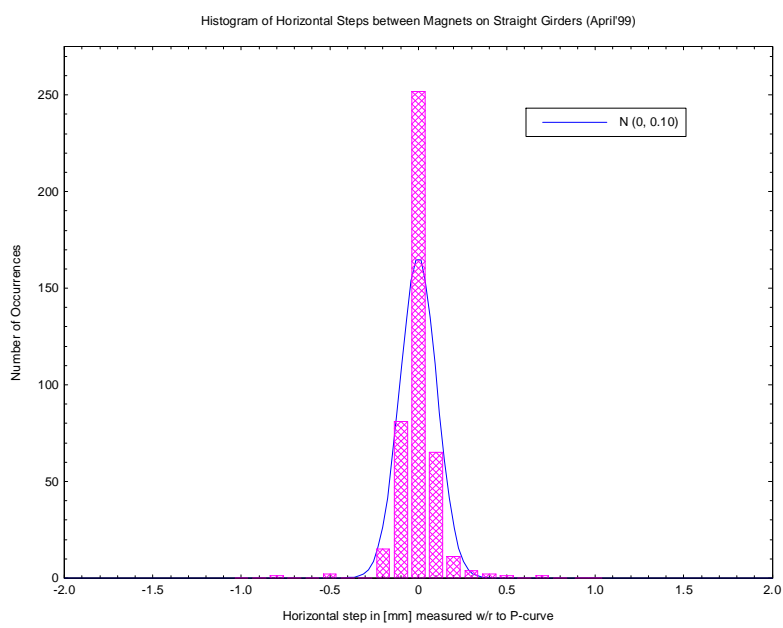

Fig 11 Transverse magnet step data, 1998

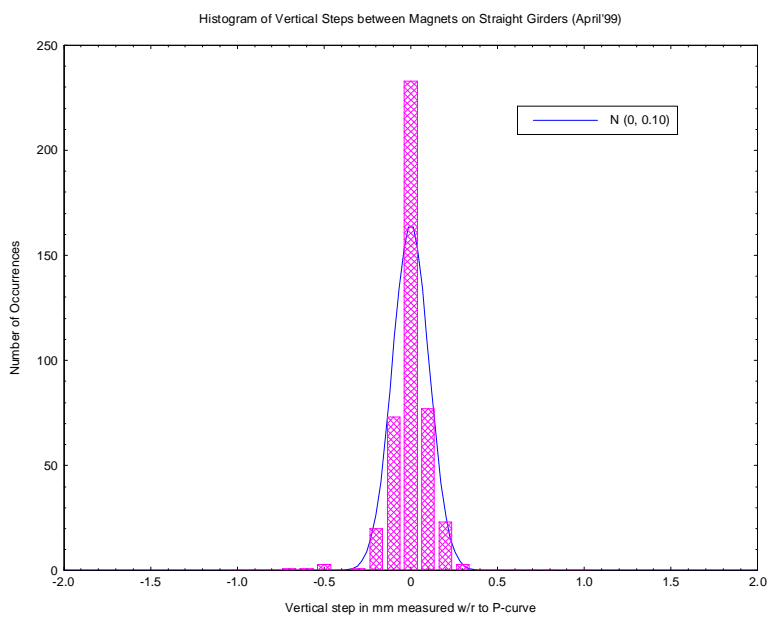

Fig 13 Vertical magnet step data, 1998 


\subsection{Inter-Girder Alignment Comparison}

The results of the inter-girder alignment are shown in Figures 14 through 17. Again the size of the steps between adjacent girders are shown in the form of histograms sorted in bins $0.1 \mathrm{~mm}$ wide. It is apparent that the majority of the alignment deterioration is occuring between girders. The standard deviation for the transverse case has increased from \pm 0.14 in 1995 to $\pm 0.40 \mathrm{~mm}$ in 1998 , while in the vertical case the standard deviation increased from $\pm 0.10 \mathrm{~mm}$ in 1995 to $\pm 0.20 \mathrm{~mm}$ in 1998 . In both cases many data points exceed the $2 \sigma$ limit. The transverse alignment shows much worse deterioration than the vertical case.

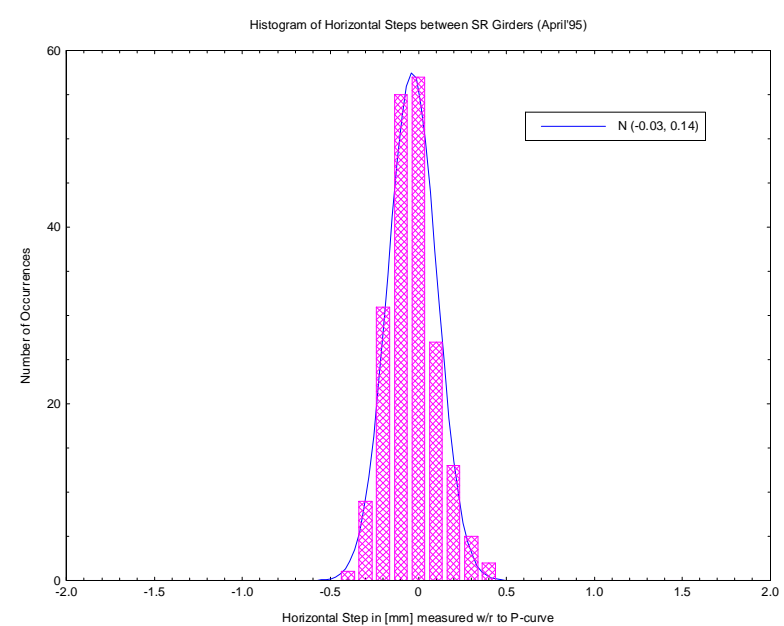

Fig 14 Transverse girder step data, 1995

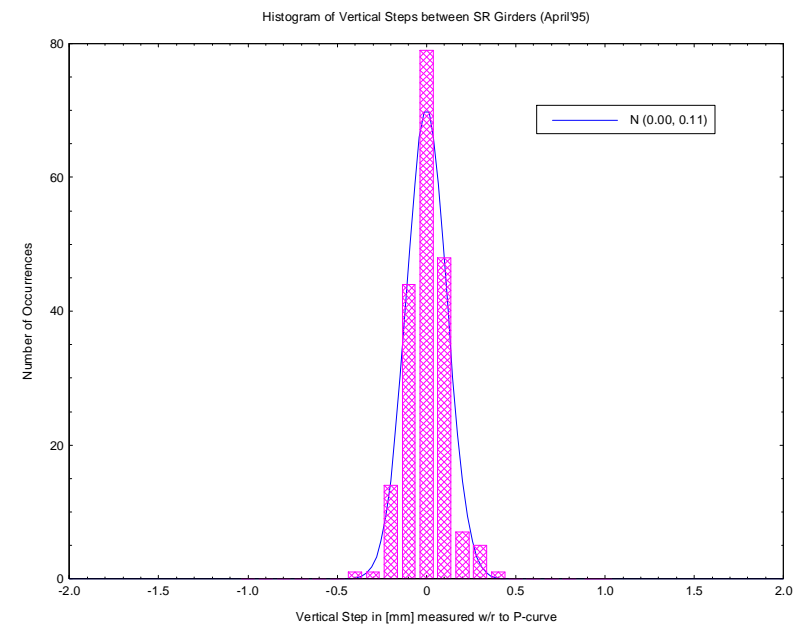

Fig 16 Vertical girder step data, 1995

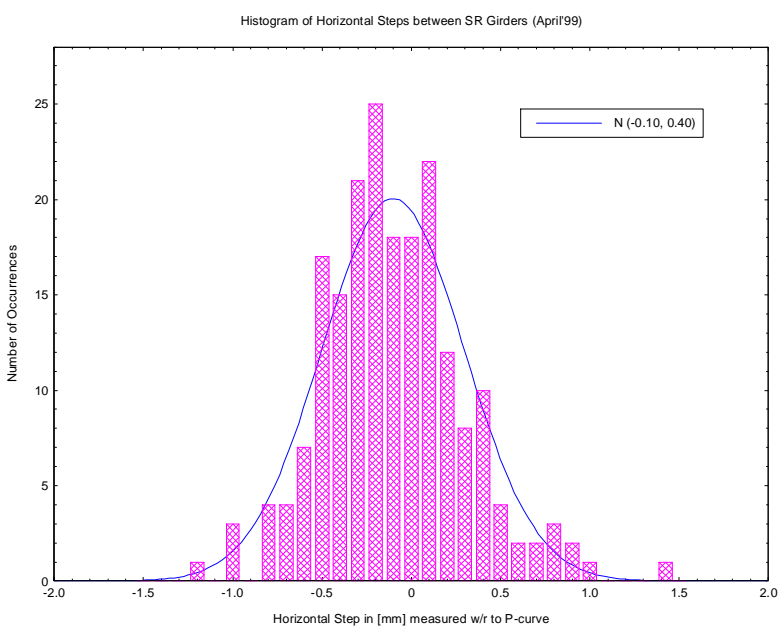

Fig 15 Transverse girder step data, 1998

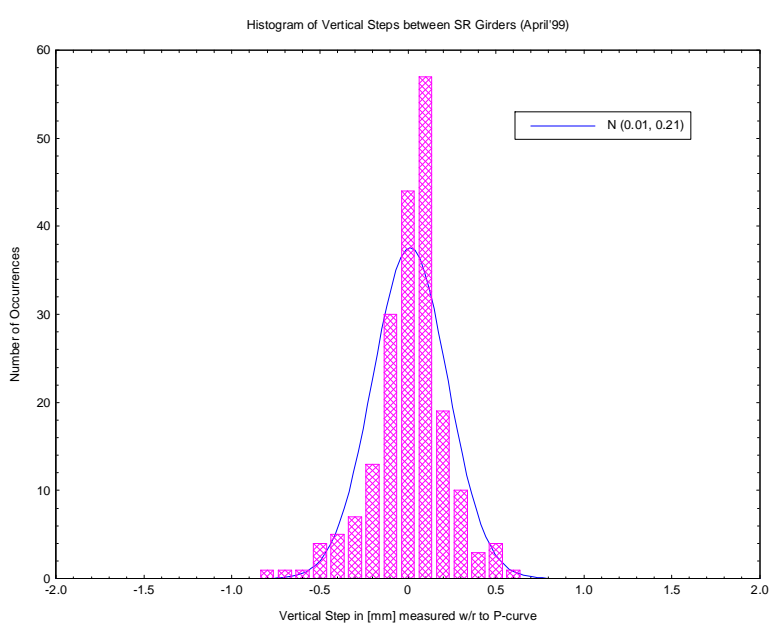

Fig 17 Vertical girder step data, 1998 


\section{SUMMARY}

As one would expect, the alignment of the APS storage ring is deteriorating over time. By separating the data into intra- and inter-girder subsets, we were able show that the main source for the alignment deterioration can be attributed to changes in the relative girder alignment. The driving force for these misplacements is still under investigation. Some possible causes for the transverse motion have been suggested:

1. Temperature fluctuations in the storage ring could cause girder creep over time.

2. The girders are virtually floating on vibration damping pads [9]. Changes in the viscosity of these pads could be the cause for the transverse motion.

3. Floor settlements, transverse to the beam direction, induce roll in the girders that is seen as transverse motion.

Our main focus has always been directed towards large changes along the vertical direction. This is still a proper assumption. However, settlements affect the string of girder supports not only along the beamline but also perpendicular to that line. This kind of settlement will affect the girder roll and therefore the transverse position. Due to the unfavorable long lever arm from the floor support to the beam height, any small transverse settlement is amplified and produces a large transverse motion. As a result, future synchrotron light sources would be best served to minimize the distance from the floor to the beam height. This type of construction has the additional advantage of reducing vibration effects, which may render vibration-damping pads unnecessary.

We are currently in the process of mapping the APS storage ring a third time in order to follow the trend of distortions seen so far. We have started to take roll measurements in the areas showing the largest steps in order to verify the assumption made in paragraph three of the above list of possible causes. However, based on this small sample, the results are inconclusive.

The roll measurements are taken using special roll fixtures. In hindsight it would be better to have at least three fiducial points per magnet in order to track these components as three-dimensional rigid bodies. This would increase the time for fiducialization but would make the mapping process, especially when using laser tracker systems, much faster. This feature is especially valuable when considering the short time periods the accelerator components are accessible for survey.

Furthermore it would have been beneficial if all multipoles had been located on one girder, leaving the dipole on its own support system. Dipoles usually have a more relaxed position tolerance compared to the multipole requirements. However, dipole roll settings are usually more stringent. A separation of these two magnet types would have better facilitate these different requirements. 


\section{ACKNOWLEDGMENT}

I would like to acknowledge all APS survey and alignment personnel for their efforts in collecting the data and handling the analysis of these large data sets as well as Cathy Eyberger for her helpful suggestions while editing and refining this paper.

\section{REFERENCES}

[1] Advanced Photon Source RESEARCH, Number 2, June 1999 (ANL/APS/TB-36).

[2] H. Friedsam, The Alignment of the Advanced Photon Source at Argonne National Laboratory, Proceedings of the Third International Workshop on Accelerator Alignment, Annecy, France, September 1993, pp. I/1 - I/8.

[3] 7-GeV Advanced Photon Source Conceptual Design Report, Argonne National Laboratory, ANL-87-15, 1987.

[4] H. Friedsam, A New Accelerator Alignment Concept using Laser Trackers, Proceedings of the Fourth European Particle Accelerator Conference, London, June 1994, pp. 2567 - 2570.

[5] H. Friedsam, M. Penicka, S. Zhao, Beamline Smoothing of the Advanced Photon Source, Proceedings of the 1995 Particle Accelerator Conference, Dallas, USA, March 1995, pp. 2084 - 2086.

[6] E. Lessner, E. Crosbie, S. Milton, Effects of the Vertical Girder Realignment in the Argonne APS Storage Ring, Proceedings of the 1999 Particle Accelerator Conference, New York, USA, 1999 (to be published).

[7] H. Friedsam, M. Penicka, J. Error, Status Report on the Long-Term Stability of the Advanced Photon Source, Proceedings of the Fifth International Workshop on Accelerator Alignment, Argonne, Illinois, October 1997 (CD-ROM).

[8] G. Decker, O. Singh, H. Friedsam, J. Jones, M. Ramanathan, D. Shu, Reduction of X-BPM Systematic Errors by Modification of Lattice in the APS Storage Ring, Proceedings of the 1999 Particle Accelerator Conference, New York, USA, 1999 (to be published).

[9] D. Mangra, S. Sharma, J. Jendrzejczyk, Passive vibration damping of the APS machine components, Proceedings of the Conference on Synchrotron Radiation Instrumentation '95, Argonne, Illinois, October 1995, Rev. Sci. Instrum., 67 (9), September 1996 (CD-ROM). 